\title{
Improved Electrical Properties of Indium Gallium Zinc Oxide Thin- Film Transistors by AZO/Ag/AZO Multilayer Electrode
}

\author{
Young-Soo No ${ }^{1,2}$ Jeong-Do Yang ${ }^{3}$, Dong-Hee Park ${ }^{1}$, Tae-Whan Kim² ${ }^{2}$ Ji-Won Choi ${ }^{4}$, and Won-Kook Choi ${ }^{1,+}$
}

\begin{abstract}
We fabricated an a-IGZO thin film transistor (TFT) with AZO/Ag/AZO transparent multilayer source/drain contacts by rf magnetron sputtering. a-IGZO TFT with AZO/Ag/AZO multilayer S/D electrodes (W/L $=400 / 50 \mu \mathrm{m})$ showed a subs-threshold swing of 3.78 $\mathrm{V} / \mathrm{dec}$, a minimum off-current of $10^{-12} \mathrm{~A}$, a threshold voltage of $0.41 \mathrm{~V}$, a field effect mobility of $10.86 \mathrm{~cm}^{2} / \mathrm{Vs}$, and an on/off ratio of $9 \times$ $10^{9}$. From the ultraviolet photoemission spectroscopy, it was revealed that the enhanced electrical performance resulted from the lowering of the Schottky barrier between a-IGZO and Ag due to the insertion of an AZO layer and thus the AZO/Ag/AZO multilayer would be very appropriate for a promising S/D contact material for the fabrication of high performance TFTs.
\end{abstract}

Keywords : a-IGZO TFT, AZO/Ag/AZO transparent electrode, Field effect mobility, Schottky barrier

\section{INTRODUCTION}

Recently, transparent-oxide-semiconductor-based transistors have shown much attention in application such as active-matrix organic light-emitting displays (AMOLED), active-matrix liquid-crystal displays (AMLCDs), and flexible displays [1-3]. Thin film transistors (TFTs) using oxide-semiconductor channels have been intensively investigated because oxide semiconductors such as amorphous In-Ga-Zn-O ( $a$-IGZO) can be formed at a low-temperature process and show larger motilities than those of hydrogenated amorphous silicon TFTs [4].

One of the essential issues to be considered for realizing the improved performance is the influence of source/drain (S/D) electrodes, including materials, contact quality, and parasitic resistance. In general, heavily doped regions

\footnotetext{
${ }^{1}$ Interface Research Center, Korea Insitute of Science and Technology, Sungbuk Gu, Hwarnagno 14 gil 5, Seoul 136-791, Korea

${ }^{2}$ Advanced Semiconductor Research Center, Division of Electrical and Computer Engineering, Hanyang University, 17 Haengang-Dong, Seongdong-Gu, Seoul 133-791, Korea

${ }^{3}$ Department of Physics, Yonsei University, Seoul 120-749, Korea

${ }^{4}$ Electronic Materials Research Center, Korea Insitute of Science and Technology, Sungbuk Gu, Hwarnagno 14 gil 5, Seoul 136-791, Korea ${ }^{+}$Corresponding author: wkchoi@kist.re.kr

(Received : Feb. 18, 2013, Revised : Mar. 13, 2013, Accepted : Mar. 14, 2013)
}

This is an Open Access article distributed under the terms of the Creative Commons Attribution Non-Commercial License(http://creativecommons.org/licenses/by$\mathrm{nc} / 3.0$ )which permits unrestricted non-commercial use, distribution, and reproduction in any medium, provided the original work is properly cited. below S/D electrodes were adopted to obtain good contact properties. Attempts to dope the selected contact regions of $a$-IGZO films by Ar plasma treatment have proven significant improvement in device performance [5]. However, this doping process possibly damages the film surface by sputtering ion $\left(\mathrm{Ar}^{+}\right)$bombardment. Barquinha et al.[6] reported that various $\mathrm{S} / \mathrm{D}$ electrodes affected the drain current of TFTs and that an annealing process improved the electrical properties. Park etal.[7] investigated the effects of $\mathrm{S} / \mathrm{D}$ series resistance and transfer length on $a$-IGZO TFTs. So far, indium tin oxide (ITO), Au, Al, indium zinc oxide (IZO), and $\mathrm{Pt} / \mathrm{Ti}$ were used as $\mathrm{S} / \mathrm{D}$ electrodes for a-IGZO TFTs [5, 8, 9]. Although oxide based electrodes, such as ITO and IZO electrodes allow fully transparent TFTs, their large electron affinity of the oxide electrodes (and $\mathrm{Au}$ ) causes non-ohmic behaviors in the linear regime of output characteristics due to the formation of the Schottky-like barrier between the electrodes and the $a$-IGZO films [9]. X. Zou etal. also reported that $\mathrm{AZO} / \mathrm{Al}$ heterojunction $\mathrm{S} / \mathrm{D}$ contacts showed the forming of a good ohmic contact and the improved mobility and the subthreshold gate voltage swing. Furthermore, the ohmic contact properties could be improved by replacing electrode materials and/or by modifying the contact area. Among many efforts to develop new TCO materials, the multilayer of dielectric material/metal/dielectric material (DMD) has been suggested as one of candidates to overcome the limit in electrical resistivity of single layer TCOs ever reported 
because it allows both the overall carrier concentration and the mobility to be increased, prevailing to some extent the limitation imposed by ionized impurity scattering in metaloxide single layers and can increase transparency in the visible range of the wavelength due to the anti-reflection principle of successive layer coating for $\operatorname{high}\left(\mathrm{n}_{1}\right) /$ low $\left(\mathrm{n}_{2}\right) /$ high refractive index $\left(\mathrm{n}_{1}\right)$ [10]. Among the most widely studied dielectric films in DMD structures such as $\mathrm{Al}$ doped $\mathrm{ZnO}$, Ga doped $\mathrm{ZnO}$ and ITO, Al-doped $\mathrm{ZnO}$ (AZO) thin film has become a good alternative for transparent electrode applications, due to its low resistivity on the order of $10^{-4} \Omega \cdot \mathrm{cm}$, inexpensive source materials, and high transpar-ency in the near UV and visible range.

This study investigates novel multilayer AZO/Ag/AZO $\mathrm{S} / \mathrm{D}$ contacts to enhance the performance of $a$-IGZO TFTs. The mobility, on/off current ratio and sub-threshold gate voltage swing of the $a$-IGZO TFTs with AZO/Ag/AZO layer S/D electrodes are investigated. The improved mobility and the sub-threshold gate voltage swing of the $a$ IGZO TFT with AZO/Ag/AZO S/D contacts compared to the TFTs with single-Ag S/D electrodes are elucidated by carefully examining the variation of potential barriers at the interface through ultraviolet photoemission spectroscopy (UPS).

\section{EXPERIMENTAL}

Fig. 1 shows a schematic cross-sectional view of the TFT structure. This was fabricated on the p-type boron doped silicon wafer with low resistivity $(\rho \sim 0.001 \Omega \cdot \mathrm{cm}$, as the gate electrode. $\mathrm{SiO}_{2}(200 \mathrm{~nm})$ thin film as an insulating layer was thermally grown on the Si substrate by a chemical vapor deposition. These substrates were then cleaned by acetone, methanol, ethanol and de-ionized water in an ultrasonic bath. Thin films of $a$-IGZO (40 nmthick) were deposited as the active layer using rf magnetron sputtering with sintered $\mathrm{InGaZnO}_{4}\left(\mathrm{In}_{2} \mathrm{O}_{3}: \mathrm{Ga}_{2} \mathrm{O}_{3}: \mathrm{ZnO}\right.$ $=1: 1: 199.999 \%$, purity) as the target material.

The initial vacuum level is lower than $1 \times 10^{-4} \mathrm{~Pa}$. Presputtering was performed to remove any contamination on the target surface for $10 \mathrm{~min}$ prior to the deposition of the active layer. IGZO thin films were sputtered at room temperature in an argon atmosphere with an oxygen partial pressure of $5 \%$ corresponding to the working pressure of $0.67 \mathrm{~Pa}$. The active layer was patterned by conventional photolithography and a lift-off process. The post-annealing process was carried out in pure $\mathrm{O}_{2}$ ambient using RTA equipment at $300^{\circ} \mathrm{C}$. The $\mathrm{S} / \mathrm{D}$ electrodes were defined using a lift off process. To form the top S/D contact of Ag and $\mathrm{AZO} / \mathrm{Ag} / \mathrm{AZO}$ multilayers were deposited by rf magnetron sputtering. The Ag layer with a nominal 100nm was deposited on the patterned $a$-IGZO film by RF sputtering with an Ar ratio of $20 \mathrm{sccm}$. For the AZO/Ag/ AZO multilayer, the bottom AZO layer was sputtered at 80 $\mathrm{W}$ rf power and $0.53 \mathrm{~Pa}$ Ar plasma gas pressure. The Ag intermediate layer was successively deposited by rf magnetron sputtering at $20 \mathrm{~W}$ and $0.53 \mathrm{~Pa}$ Ar plasma gas pressure. Then, the top AZO layer was sputtered by the same method with the AZO first layer. The resistivity and optical transmittance of the $\mathrm{AZO} / \mathrm{Ag} / \mathrm{AZO}$ multilayer electrodes were measured by Hall measurements and UV/visible spectroscopy (Model: Perkin Elmer Lambda 18), respectively. Transmission electron microscopy (TEM: FEI Tecnai F20) was employed to investigate the structure of the multilayer electrodes. The electrical characteristics of $a$-IGZO films and TFT devices were measured using a Keithley 4200 precision semiconductor parameter analyzer in the dark. Work functions of $\mathrm{Ag}$, $\mathrm{AZO} / \mathrm{Ag} / \mathrm{AZO}$ including IGZO thin films were determined from the positions the Fermi level measured from the tangent line extrapolation of the onset for the secondary electron peak in the ultraviolet photoemission spectroscopy (UPS, Kratos AXIS-NOVA) spectra. The photon energy of the UPS light source (He (I) radiation ) was $h \nu=21.2 \mathrm{eV}$ and the energy of the band pass filter in the analyzer was fixed at $20 \mathrm{eV}$.

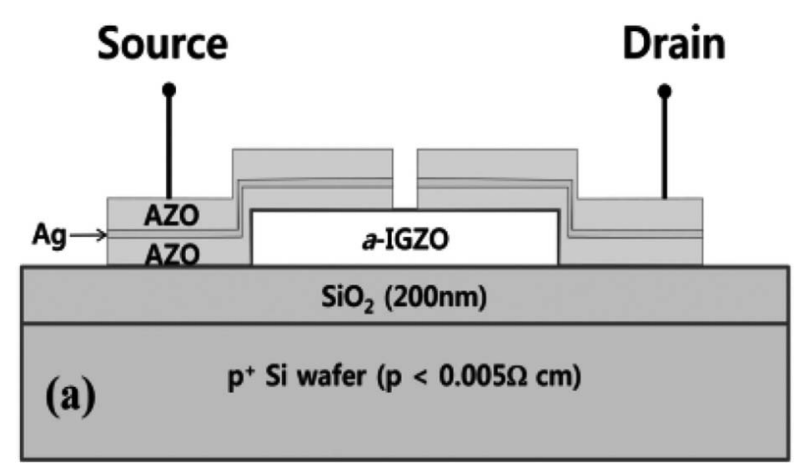

Fig. 1. Schematic diagram of the $a-\mathrm{IGZO}$ TFT with AZO/Ag/ AZO source-drain electrode.

\section{RESULTS AND DISCUSSIONS}

Fig. 2 presents the optical transmission spectra of the $\mathrm{AZO} / \mathrm{Ag} / \mathrm{AZO}$ thin film deposited on glass substrate. The 
inset in Fig. 2 shows a cross sectional TEM image of the $\mathrm{AZO} / \mathrm{Ag} / \mathrm{AZO}$ multilayer indicating that the thicknesses of each layer are approximately $47 \mathrm{~nm}$ and $11 \mathrm{~nm}$ for the AZO and Ag layer, respectively. Based upon the optical simulation using the Macleod program and experimental data, we carefully determined the optimal thickness of $\mathrm{Ag}$ and $\mathrm{AZO}$ thin films and tried to deposit at this condition. The AZO/Ag/AZO thin film shows the electrical resistivity $\rho=8.6 \times 10^{-5} \Omega \cdot \mathrm{cm}$, carrier concentration $\mathrm{n}_{\mathrm{e}}=8.0 \times$ $1022 / \mathrm{cm}^{3}$, and mobility $\mu=20 \mathrm{~cm}^{2} / \mathrm{Vs}$.

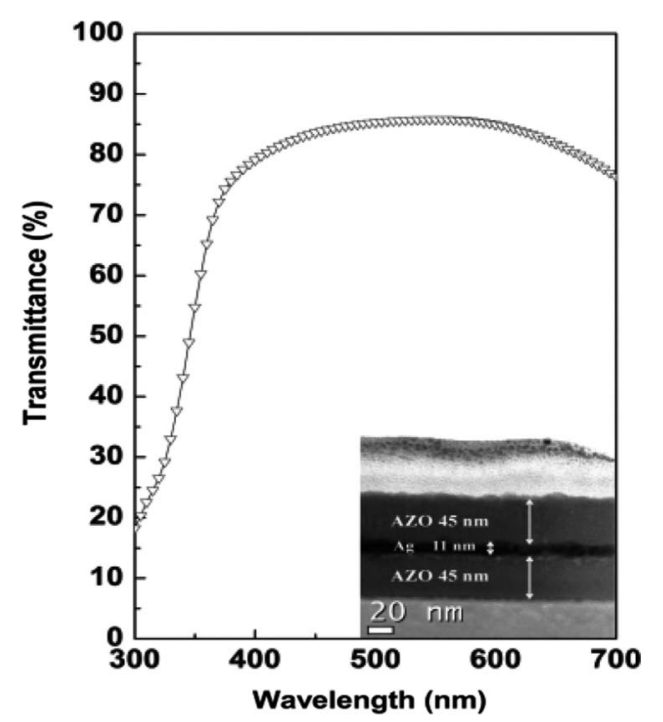

Fig. 2. Optical transmission spectra of the AZO/Ag/AZO films. The inset shows the cross sectional view of the transmission electron microscope (TEM) image of $\mathrm{AZO} / \mathrm{Ag} / \mathrm{AZO}$ films.

Average transmittance of $\mathrm{AZO} / \mathrm{Ag} / \mathrm{AZO}$ thin film is approximately $85 \%$ in the visible wavelength of $400-650 \mathrm{~nm}$.

The typical output characteristics of $a$-IGZO TFTs with single-Ag or AZO/Ag/AZO S/D electrodes are depicted in Fig. 3. It can be seen from Fig. 3 that the saturation current for the $a$-IGZO TFT with AZO/Ag/AZO S/D electrodes is $45.5 \mu \mathrm{A}$ at a bias of $\mathrm{V}_{\mathrm{DS}}=\mathrm{V}_{\mathrm{GS}}=20 \mathrm{~V}$, while the TFT with $\mathrm{Ag}$ electrodes shows a drain current of $1.7 \times 10^{-3} \mu \mathrm{A}$ at the same drain and gate bias voltage, indicating that a multilayer electrode greatly increases the driving current by improving the S/D contact. The increased drain current of the $a$-IGZO TFT with AZO/Ag/AZO multilayer electrodes can be understood in the same manner as the conventional metal oxide-semiconductor transistor structures, which is attributed the reduction of the parasitic contact resistance between the electrode and the channel layer [11]. Furthermore, the output curves of the $a$-IGZO TFT with
Ag electrodes show obvious current crowding at low $\mathrm{V}_{\mathrm{DS}}$ $(<2.5 \mathrm{~V})$, whereas the drain current of the $a$-IGZO TFT with $\mathrm{AZO} / \mathrm{Ag} / \mathrm{AZO}$ multilayer electrodes shows a steep rise in the low $\mathrm{V}_{\mathrm{DS}}$ region, which further indicates the low contact resistivity of the $\mathrm{AZO} / \mathrm{Ag} / \mathrm{AZO} / \mathrm{IGZO}$ structure. The transfer characteristics of the $a$-IGZO TFTs $(\mathrm{W} / \mathrm{L}=400 / 50 \mu \mathrm{m})$ are shown in Fig. 4.
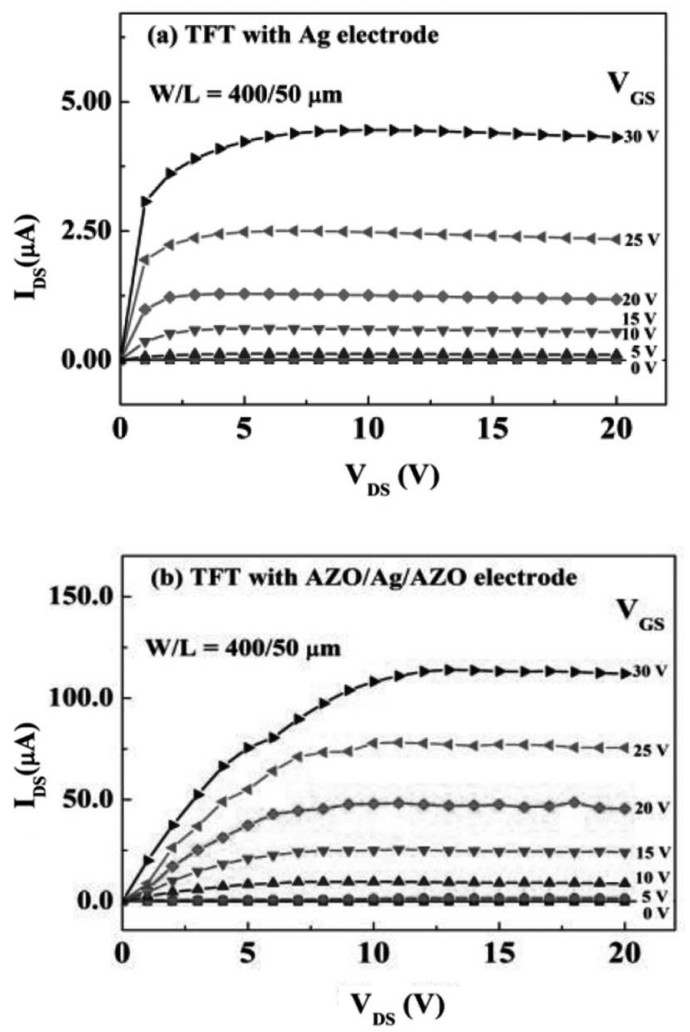

Fig. 3. Output characteristics of the $a$-IGZO TFT (W/L $=400 / 50$ $\mu \mathrm{m}$ ); (a) with $\mathrm{Ag}$ electrodes and (b) with $\mathrm{AZO} / \mathrm{Ag} / \mathrm{AZO}$ multilayer electrodes.

An on-off current ratio of $9.1 \times 10^{9}$ at $10 \mathrm{~V}$ for the TFT with $\mathrm{AZO} / \mathrm{Ag} / \mathrm{AZO}$ electrodes can be achieved, which is two orders of magnitude larger than that of the TFT with Ag electrodes $\left(3.4 \times 10^{7}\right)$. The minimum OFF-state current mainly attributed to the gate leakage current lies in the range of $\sim 10^{-11} \mathrm{~A}$ and is also favorable to reduce the power consumption, because the operating TFTs for display spends most of their duty cycle in the OFF-state. Under these conditions, the field-effect mobility $\left(\mu_{\mathrm{FE}}\right)$ is calculated using the following equation (1);

$$
\mu_{\mathrm{FE}}=(\mathrm{L} / \mathrm{W})\left(\mathrm{g}_{\mathrm{m}} / \mathrm{C}_{\mathrm{o}} \mathrm{V}_{\mathrm{DS}}\right)
$$


where $\mathrm{C}_{\mathrm{o}}$ denotes the insulator capacitance, $\mathrm{W} / \mathrm{L}$ denotes the aspect ratio of the device, and $\mathrm{g}_{\mathrm{m}}$ denotes the transconductance $\left(\mathrm{g}_{\mathrm{m}}=\partial \mathrm{I}_{\mathrm{DS}} / \partial \mathrm{V}_{\mathrm{GS}}\right)$.

The field-effect mobility ( $\mu_{\mathrm{FE}}$ ) of the $a$-IGZO TFT with $\mathrm{AZO} / \mathrm{Ag} / \mathrm{AZO}$ layer S/D electrodes is calculated as 10.8 $\mathrm{cm}^{2} / \mathrm{Vs}$, which is higer than that of $1.16 \mathrm{~cm}^{2} / \mathrm{Vs}$ of the TFT with Ag electrodes. The threshold voltage $\left(\mathrm{V}_{\text {th }}\right)$ was derived from a linear fitting to a $\left(\mathrm{I}_{\mathrm{DS}}\right)^{1 / 2}$ versus VGS plot using the equation

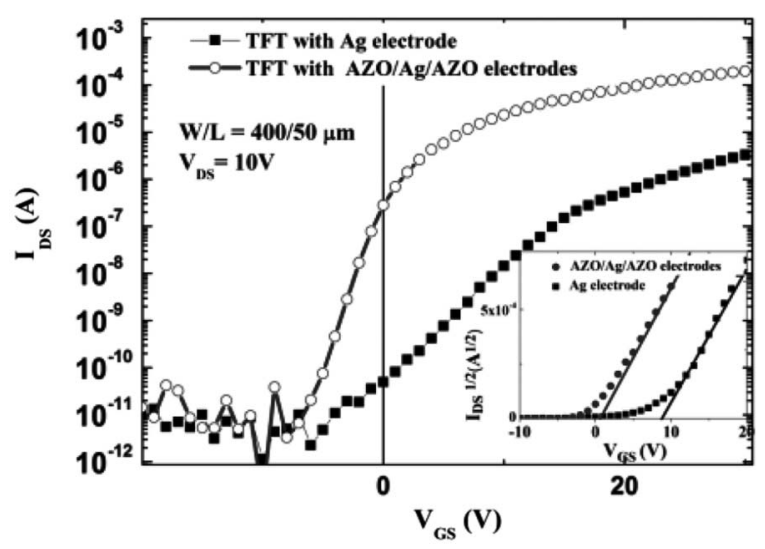

Fig. 4. Transfer characteristics of the a-IGZO TFT with a W/L = $400 / 50 \mu \mathrm{m}$. The inset indicates the $\mathrm{I}_{\mathrm{DS}}{ }^{1 / 2}-\mathrm{V}_{\mathrm{GS}}$ at $\mathrm{V}_{\mathrm{DS}}=$ $10 \mathrm{~V}$.

$\mathrm{I}_{\mathrm{DS}}=\left(\mu \varepsilon_{\mathrm{o}} \mu \varepsilon_{\mathrm{r}} \mathrm{W} / 2 \mathrm{Ld}\left(\mathrm{V}_{\mathrm{GS}}-\mathrm{V}_{\text {th }}\right)^{2}\right.$, where $\mathrm{W}$ is the channel width, $\mathrm{L}$ is the channel length, $\varepsilon_{0}$ is the free-space permitivity, $\varepsilon_{\mathrm{r}}$ is the relative dieclectric constant of the gate insulator, and $\mathrm{d}$ is the thickness of the gate insulator. The threshold voltages are extracted as 7.73 and $0.41 \mathrm{~V}$ for $a$ IGZO with Ag electrodes and AZO/Ag/AZO electrodes, repectively. A reliable pinch-off at zero gate bias volage can be found for the $a$-IGZO TFT with AZO/Ag/AZO layer S/D electrodes, while the $\mathrm{I}_{\mathrm{DS}}$ of $\sim 10^{-9} \mathrm{~A}$ at a zero bias for the TFT with Ag electrodes should increase the power consumption. The subthreshold gate voltage swing (SS) can be extracted using the following equation at $\mathrm{V}_{\mathrm{DS}}=$ const.:

$$
\mathrm{SS}=\partial \mathrm{V}_{\mathrm{GS}} / \partial\left(\log \mathrm{I}_{\mathrm{DS}}\right)
$$

The SS is estimated to be about 7.83 and $3.78 \mathrm{~V} \mathrm{dec}^{-1}$ for TFTs with Ag electrodes and AZO/Ag/AZO electrodes, respectively. The $a$-IGZO TFT with $\mathrm{AZO} / \mathrm{Ag} / \mathrm{AZO}$ layer $\mathrm{S} / \mathrm{D}$ electrodes can be found to improve subthreshold swing compared to the TFT with Ag S/D electrodes, even though the TFTs have identical materials and preparation process for the channel and gate dielectric, which should be attributed to an increased driving current resulting from a decreased S/D contact resistance.

The TLM was adopted to evaluate the contact resistance of different electodes [12]. By defining the total TFT ONn resistance (RT) as $\mathrm{RT}=\mathrm{V}_{\mathrm{DS}} / \mathrm{I}_{\mathrm{DS}}$, $\mathrm{RT}$ can be expressed as:

$$
R_{\mathrm{T}}=\mathrm{V}_{\mathrm{DS}} / \mathrm{I}_{\mathrm{DS}}=\mathrm{r}_{\mathrm{ch}} \mathrm{L}+\mathrm{R}_{\mathrm{SD}}
$$

with $\mathrm{r}_{\mathrm{ch}}=1 / \mu_{\mathrm{EF}-\mathrm{i}} \mathrm{C}_{\mathrm{OX}} \mathrm{W}\left(\mathrm{V}_{\mathrm{GS}}-\mathrm{V}_{\text {th-i }}\right)$, where rch is the channel resistance per unit channel length, $\mathrm{R}_{\mathrm{S} / \mathrm{D}}$ is the series resistance at the $\mathrm{S} / \mathrm{D}$ contacts, $\mu_{\mathrm{EF}-\mathrm{i}}$ is the intrinsic field effect mobility, $\mathrm{V}_{\text {th-i }}$ is the intrinsic threshold voltage, and $\mathrm{C}_{\mathrm{OX}}$ is the gate-insulator capacitance per unit area. Subsequently, the width-normalized $\mathrm{S} / \mathrm{D}$ resistivity $\left(\mathrm{R}_{\mathrm{SD}} \mathrm{W}\right)$ is calculated as 253 and $1.9 \mathrm{k} \Omega \mathrm{cm}$ for the $a$-IGZO TFT with $\mathrm{Ag}$ and $\mathrm{AZO} / \mathrm{Ag} / \mathrm{AZO}$ multilayer electrodes, respectively. The device structures with $\mathrm{AZO} / \mathrm{Ag} / \mathrm{AZO}$ mutlilayer electrodes are highly advantageous for improving the device performance of $a$-IGZO TFTs.

Fig. 5 (a) presents a schematic energy band diagram of individually separated layers of $\mathrm{Ag}, \mathrm{AZO} / \mathrm{Ag} / \mathrm{AZO}$ and $a$ IGZO. In the separated system, the work function of the $\mathrm{Ag}$ electrode $\left(\phi_{\mathrm{Ag}}=4.82 \mathrm{eV}\right)$ is higher than the electron affinity of a-IGZO ( $\left.\phi_{\text {IGZO }}=4.25 \mathrm{eV}\right)$ calculated from the UPS method. The work function data are obtained from the following equation,

$$
\phi=h \nu(21.2 \mathrm{eV})-\mathrm{E}_{\text {cutoff }}+{ }_{\mathrm{x}} \mathrm{E}_{\mathrm{F}}
$$

where $\phi, \mathrm{E}_{\text {cutoff }}$ and $\mathrm{E}_{\mathrm{F}}$ are the work function, secondary electron cutoff and Fermi level, repsectively.

The work functions were determined from the positions the of Fermi level measured from the tangent line extrapolation of the onset regarding the secondary electron peak in the spectra. Therefore, when the Ag electrode is brought into direct contact with an $a$-IGZO channel layer, a Schottky barrier is formed with the barrier height $\left(\Phi_{B}\right)$ of $0.57 \mathrm{eV}$. Such a large electron affinity of the metal linear electrode (Ag, Au and $\mathrm{Pt}$ ) causes nonlinear behaviors in the channel layer. A Schottky barrier is formed with the barrier regime of output characteristics due to the formation of electrode being brought into direct contact with the $a$ IGZO height Schottky-like barrier between the elctrodes and the $a$-IGZO films. The Ohmic contact properties can be improved by replacing elecrode materials and/or by 
modifying the contact area. The therminoic emission current $\mathrm{J}$ is formed by electrons overcoming the Schottky barrier by the thermal energy from the metal to the $a$-IGZO channel layer.

$J=A^{*} T^{2} \exp \left(-q \Phi_{B} / k_{B} T\right)\left[\exp \left(-q V / n_{B} T\right)\right]^{-1}$

where $\mathrm{A}^{*}, \mathrm{~T}, \mathrm{k}_{\mathrm{B}}, \mathrm{n}$, and $\Phi_{\mathrm{B}}$ are the Richardson constant, temperature, ideality factor, the Boltzmann's constant, and Schottky barrier height, respectively.

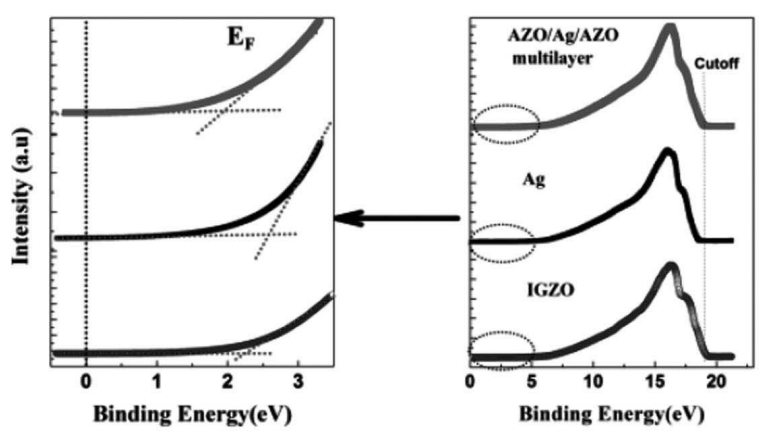

(a)

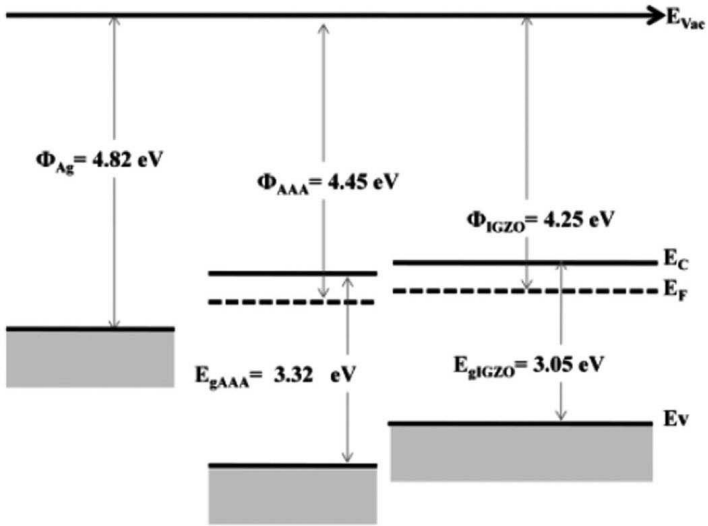

(b)

Fig. 5. (a) UPS spectra of the AZO/Ag/AZO films, Ag, and IGZO film surface. (b) Schematic energy band diagram in separated systems.

The property of contacts on the $a$-IGZO layer depends on the Schottky barrier height [12]. When the AZO/Ag/ AZO mutilayer with the barrier height $\left(\Phi_{\mathrm{B}}\right)$ of $0.02 \mathrm{eV}$ is used as source/drain contacts, we focused our interest on using a heavily doped layer as a contact to source/drain because it is the conventional technology for oxide semiconductor devices. It also allows ohmic contacts, and in oxide-based semiconductors, it reduces the possibility of forming an oxide barrier at the interface, thus reducing the probability of forming an undesirable Schottky barrier [13].

\section{CONCLUSIONS}

In summary, the electrical properties were observed to be significantly improved for the IGZO with AZO/Ag/AZO to the Ag electrode. Our results suggest that the $\mathrm{AZO} / \mathrm{Ag} / \mathrm{AZO}$ multilayer is one of promising transparent conducting electrodes to attain better performance of IGZO based TFTs in terms of both electrical and optical aspects.

\section{ACKNOWLEDGMENT}

This work was support by both KIST Future Resource Program (2E24011) and the Converging Research Center Program through the National Research Foundation of Korea (NRF) funded by the Ministry of Education, Science, and Technology (grant number 2012K001283).

\section{REFERENCES}

[1] K. Nomura, H. Ohta, A. Takagi, T. Kamiya, M. Hirano, and H. Hosono, "Room-temperature fabrication of transparent flexible thin-film transistors using amorphous oxide semiconductors", Nature, Vol. 432, pp. 488-492, 2004.

[2] K. Nomura, A. Takagi, T. Kamiya, H. Ohta, M. Hirano, and H. Hosono, "Amorphous oxide semiconductors for high-performance flexible thin-film transistors", Jpn. J. Appl. Phys., Vol. 45, pp. 4303-4308, 2006.

[3] S.-H. K. Park, C.-S. Hwang, M. Ryu, S. Yang, C. Byun, J. Shin, J.-I. Lee, K. Lee, M. S. Oh, and S. Im, "Trans-parent and photo-stable $\mathrm{ZnO}$ thin-film transistors to drive an active matrix organic-lightemitting-diode display panel", Adv. Mater., Vol. 21, pp. 678-682, 2009.

[4] H. Yabuta, M. Sano, K. Abe, T. Aiba, T. Den, H. Kumomi, K. Nomura, T. Kamiya, and H. Hosono, "High-mobility thin-film transistor with amorphous $\mathrm{InGaZnO}_{4}$ channel Fabricated by room temperature rfmagnetron sputtering”, Appl. Phys. Lett., Vol. 89, p. 112123, 2006.

[5] J.-S. Park, J. K. Jeong, Y.-G. Mo, H. D. Kim, and S.-I. Kim, "Improvements in the device characteristics of amorphous indium gallium zinc oxide thin-film transistors by Ar plasma treatment", Appl. Phys. Lett., Vol. 90, p. 262106, 2007.

[6] P. Barquinha, A. M. Vila, G. GonCalves, L. Pereira, R. 
Martins, J. R. Morante, and E. Fortunato, "GalliumIndium-Zinc-Oxide-based thin-film transistors: influence of the source/drain material", IEEE Trans. Electron Devices, Vol. 55, pp. 954-960, 2008.

[7] J. Park, C. Kim, S. Kim, I. Song, D. Kang, H. Lim, H. Yin, R. Jung, and E. Lee, "Source/drain seriesresistance effects in amorphous gallium-indium zincoxide thin film transistors", IEEE Electron Device Lett., Vol. 29, pp. 879-881, 2008.

[8] W. Lim, S. Kim, Y.-L. Wang, J. W. Lee, D. P. Norton, S. J. Pearton, F. Ren, and I. I. Kravchenko, "Highperformance indium gallium zinc oxide transparent thin-film transistors fabricated by radio-frequency sputtering", J. Electrochem. Soc., Vol. 155, pp. H383$\mathrm{H} 385,2008$.

[9] J. H. Na, M. Kitamura, and Y. Arakawa, "High fieldeffect mobility amorphous InGaZnO transistors with aluminum electrodes", Appl. Phys. Lett., Vol. 93, p. 063501, 2008.
[10] J. D. Yang, S. H. Cho, T. W. Hong, D. I. Son, D. H. Park, K. H. Yoo, and W. K. Choi, "Organic photovoltaic cells fabricated on a $\mathrm{SnO}_{\mathrm{x}} / \mathrm{Ag} / \mathrm{SnO}_{\mathrm{x}}$ multilayer transparent conducting electrode", Thin Solid Films, Vol. 520, pp. 6215-6220, 2012.

[11] S. Luan and G.W. Neudeck, "An experimental study of the source/drain parasitic resistance effects in amorphous silicon thin film transistors", J. Appl. Phys., Vol. 72, pp. 766-772, 1992.

[12] Y. Shimura, K. Nomura, H. Yanagi, T. Kamiya, M. Hirano, and H. Hosono, "Specific contact resistances between amorphous oxide semiconductor In-Ga-Zn-O and metallic electrodes", Thin Solid Films, Vol. 516, pp. 5899-5902, 2008.

[13] C. Avis, S. H. Kim, J. H. Hur, J. Jang, and W. I. Milne, "Coplanar $\mathrm{ZnO}$ thin-film transistor using boron ion doped source/drain contacts", Electrochem. Solid State Lett., Vol. 12, pp. J93-J95, 2009. 